\title{
Massive Thymic Hyperplasia Mimicking Anterior Mediastinal Neoplasia in Occult Graves' Disease
}

\author{
Christopher James Kennedy ${ }^{1}$ David James William Paton ${ }^{2}$ \\ ${ }^{1}$ Department of Cardiothoracic Anaesthetics, Fiona Stanley Hospital, \\ Perth, Western Australia, Australia \\ ${ }^{2}$ Department of Anatomical Pathology, PathWest Tissue Pathology, \\ Perth, Western Australia, Australia \\ Address for correspondence David James William Paton, MBChB, \\ Department of Anatomical Pathology, PathWest Tissue Pathology, 11 \\ Robin Warren Drive Murdoch, Perth, Western Australia 6150, \\ Australia (e-mail: dpatonacademic@gmail.com).
}

Thorac Cardiovasc Surg Rep 2019;8:e24-e26.

Abstract
Keywords
- thymus
- thoracic surgery
- pathology

\section{Introduction}

Thymic hyperplasia is a recognized complication of established Graves' disease that can present radiologically as an anterior mediastinal mass. In this setting, thymic enlargement is expected to resolve with treatment of hyperthyroidism, and therefore imaging surveillance may be appropriate without the need to pursue invasive tissue diagnosis or therapeutic thymectomy. Recognition of the association of the reactive nature of the thymic enlargement occurring secondary to the underlying Graves' disease is crucial in avoiding unnecessary surgical intervention.

\section{Case Description}

A 24-year-old female was referred to our institution for management of an anterior mediastinal mass identified incidentally during thoracic computed tomography performed in the hours following trauma. On clinical review she described a history of several months of worsening exertional dyspnoea, with anxiety and tremor. There was no other significant medical history. Systemic review, clinical examination, and routine laboratory studies were otherwise remarkable only for a sinus tachycardia of 110 beats per minute. Review of the thoracic imaging showed a $45 \times 26 \times 34 \mathrm{~mm}$ mediastinal

received

February 13, 2019

accepted

March 13, 2019 mass with extension to the pulmonary trunk but without evidence of pericardial invasion (-Fig. 1). The appearances were suspicious for primary thymic neoplasia. Thymectomy proceeded via midline sternotomy and en-bloc resection. Intraoperatively, persistent sinus tachycardia and hypertension were noted as the thymectomy proceeded. Urgent thyroid function studies, which had not been undertaken preoperatively, showed free T4 of 50 (normal: 9-19 pmol/L) and thyroid stimulating hormone (TSH) level less than 0.01 (normal: 0.40 $4.00 \mathrm{mU} / \mathrm{L}$ ), and a diagnosis of thyrotoxicosis was made. Immediate treatment was initiated with intravenous metoprolol. Detailed postoperative review revealed mild tremor and mild proptosis without ophthalmoplegia. Serology showed TSH receptor antibody of 5.3 (normal: $<1.8 \mathrm{U} / \mathrm{L}$ ), and a diagnosis of Graves' disease was made. Retrospective radiological review of the thoracic imaging did not identify significant enlargement of the imaged thyroid. The patient was commenced postoperatively on treatment with oral propranolol and carbimazole, and was chemically and clinically euthyroid at six month follow-up.

At macroscopic examination the removed thymus was lobulated and firm, measuring $124 \times 110 \times 25 \mathrm{~mm}$ (-Fig. 2) with a weight of $87 \mathrm{~g}$ (expected normal weight for age $21.6 \pm 19.0 \mathrm{~g}^{1}$ ). Sectioning showed a uniform lobulated cream, fibrous cut surface with minimal admixed fat
License terms Stuttgart · New York

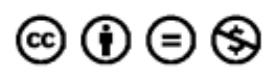




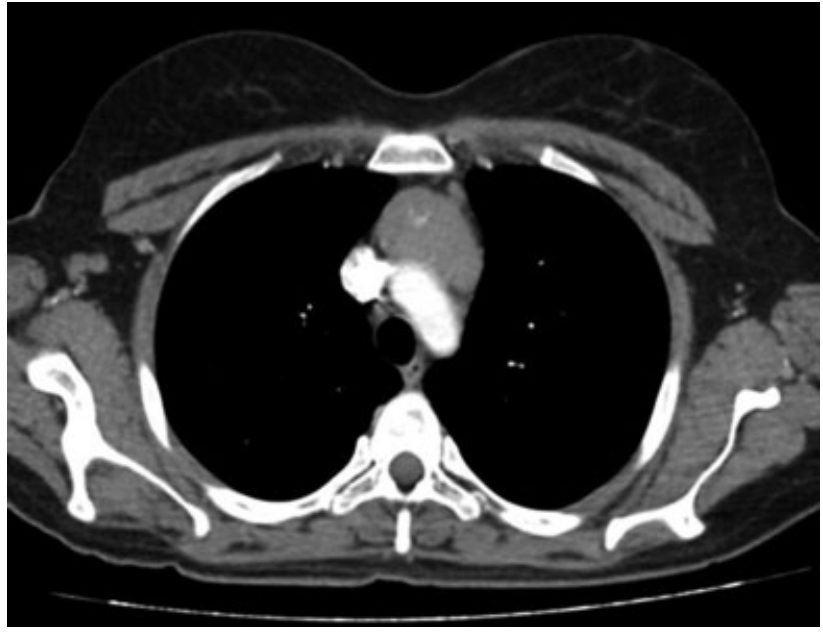

Fig. 1 Axial section of thoracic computed tomography showing anterior mediastinal mass.

and with no discrete mass. Following extensive sampling, histopathological examination revealed expanded thymic tissue consistent with "true" thymic hyperplasia (-Fig. 3A) with a minor component of "lymphoid" hyperplasia (-Fig. 3B). A clinicopathological diagnosis of thymic hyperplasia secondary to previously undiagnosed Graves' disease was therefore rendered.

\section{Discussion}

Anterior mediastinal masses are encountered uncommonly in routine clinical practice. They incite a broad but relatively

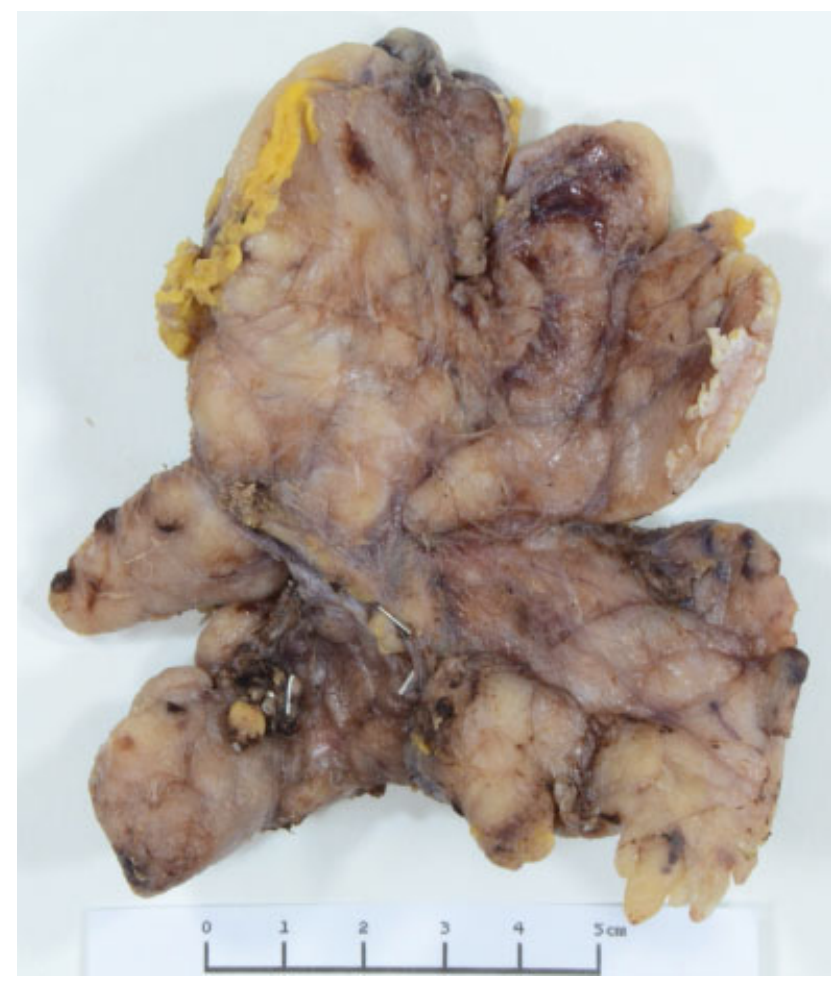

Fig. 2 Thymectomy specimen showing a measuring $124 \times 110$ $\times 25$ mm lobulated cream surface without a discrete mass. well-defined differential diagnosis which includes retrosternal thyroid and parathyroid lesions, thymic epithelial neoplasia (thymoma and thymic carcinoma), lymphoma, germ cell tumor, and metastasis. Uncommonly such masses may occur secondary to diffuse non-neoplastic enlargement of the thymus, termed "thymic hyperplasia." In most cases, thymic hyperplasia occurs as a reactive phenomenon in the setting of systemic disease and is expected to resolve with conservative management, and therefore where it is suspected, surveillance alone may be appropriate without a requirement to pursue invasive tissue diagnosis.

Two histological types of thymic hyperplasia are described. ${ }^{1}$ "True" thymic hyperplasia always presents with an enlarged thymus gland, and histologically shows ordered expansion of the cortical and medullary compartments. This type is mostly associated with severe physiological stress, such as following chemotherapy or following cessation of corticosteroid therapy. "Lymphoid" thymic hyperplasia may or may not be associated with overall gland enlargement, and on microscopy shows an increase in B-cell-rich lymphoid follicles within the thymic parenchyma. This type is associated most notably with myasthenia gravis, but has been observed frequently in other autoimmune conditions.

Graves' disease is an autoimmune disorder characterized by the production of antibodies against the thyroid stimulating hormone receptor. This leads to unopposed thyroid stimulation and enlargement with excess thyroid hormone production. There is a curious association between Graves' disease and thymic hyperplasia which has been reported by multiple authors over the past century. The observation that the thymic enlargement reverses with treatment of Graves' disease supports the postulation that the thymic hyperplasia occurs secondary to either direct stimulatory action by thyroid hormone itself or by stimulation by TSH receptor antibodies of TSH receptors within the thymus, although the exact mechanism by which this occurs is unclear. ${ }^{2}$

The true incidence of thymic hyperplasia in patients with Graves' disease is not known. Historically, histological abnormalities were identified in $38 \%$ of opportunistic thymic biopsies at the time of surgery in hyperthyroid patients. ${ }^{3}$ Although, the literature contains numerous case reports documenting the association, no study investigating the incidence of gross thymic enlargement in Graves' disease has been undertaken. It is likely that the phenomenon is under-recognized clinically because thymic hyperplasia is frequently asymptomatic and detailed thoracic imaging is rarely performed.

Numerous reports have described spontaneous resolution of Graves'related thymic enlargement upon the achievement of a euthyroid state. ${ }^{4}$ The time to resolution of thymic enlargement has varied between cases, but published guidance suggests there should be a period of at least 6 months between instigation of antithyroid treatment and follow-up thoracic imaging. ${ }^{4}$ Because Graves' disease is generally readily clinically apparent when it is associated with thymic hyperplasia, thymectomy is avoided in these patients. This means that there is an absence of detailed contemporary histological descriptions of the findings of thymectomy specimens in this setting. 

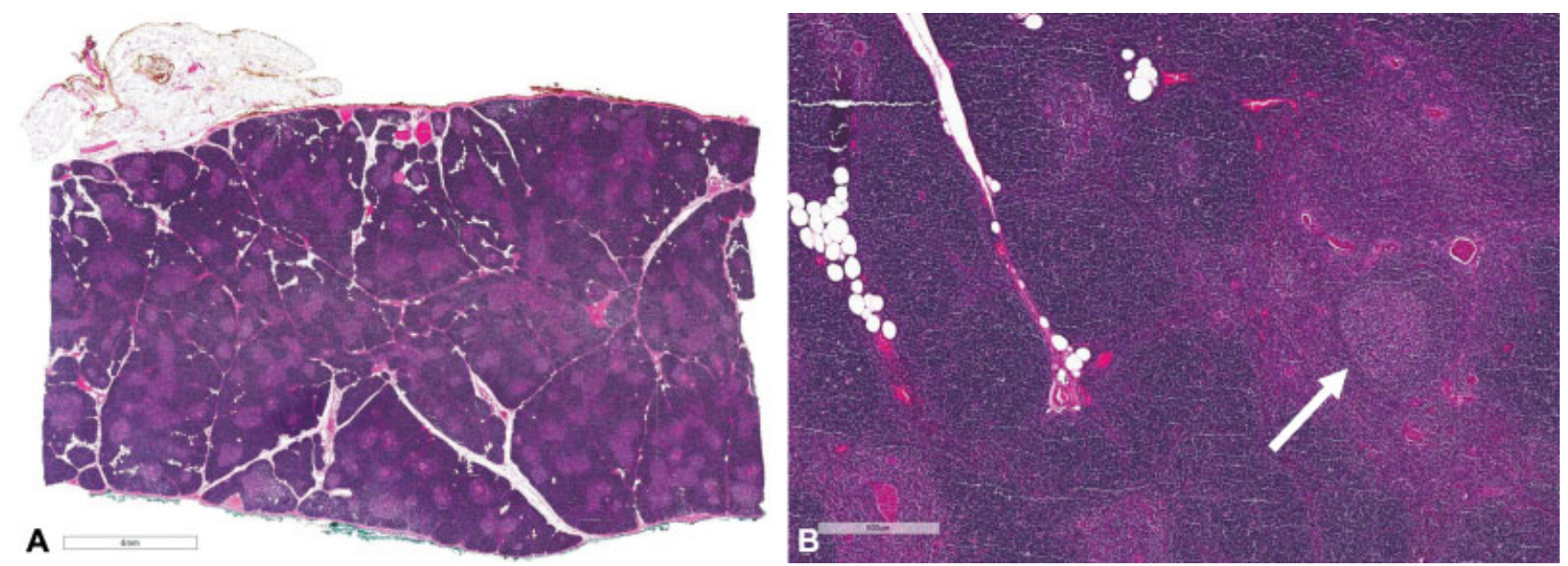

Fig. 3 (A) Low-power view of thymectomy specimen showing ordered expansion of the cortical and medullary thymic compartments in keeping with "true" hyperplasia. (B) Focal secondary lymphoid follicles in keeping with "lymphoid" hyperplasia.

We believe that this case represents the first described case of histologically confirmed massive thymic hyperplasia secondary to occult Graves' disease. Because a presumptive diagnosis of thymic neoplasia was made, we have been able to provide a rare detailed contemporary description of the histological findings in a total thymectomy specimen in the setting of untreated Graves' disease. We have described a pattern of true thymic hyperplasia superimposed upon which were minor features of lymphoid thymic hyperplasia. Both forms of hyperplasia have been described in Graves' disease, and we postulate that their cooccurrence in the current total thymectomy specimen is likely to represent a manifestation of the dual etiology of the thymic hyperplasia involving both immune and TSH-related dysregulation.

This case is a salient reminder of the frequent association of anterior mediastinal masses with systemic disease. We believe that the work-up of all anterior mediastinal masses should include a comprehensive search for medical causes of reversible thymic enlargement, and that when such potential causes exist, surveillance should be considered in suitable patients prior to surgical intervention. In addition to a detailed clinical history and examination, which aid exclusion of thymic hyperplasia due to common causes including chemotherapy, corticosteroid treatment, radiotherapy, human immunodeficiency virus and diagnosed autoimmune or connective tissue conditions, we suggest measurement of thyroid function should be performed as part of the routine approach in this setting.

\section{Conflict of Interest}

None.

\section{References}

1 Shimosato Y, Muxai K, Matsuno Y. Non-neoplastic conditions of the thymus. In: Shimosato Y, Muxai K, Matsuno Y, eds. Tumors of the Mediastinum, Armed Forces Institute of Pathology Atlas of Tumour Pathology, Series 4. Silver Spring, MD: ARP Press; 2010:271-280

2 Dalla Costa M, Mangano FA, Betterle C. Thymic hyperplasia in patients with Graves' disease. J Endocrinol Invest 2014;37(12): 1175-1179

3 Michie W, Gunn A. The thyroid, the thymus and autoimmunity. $\mathrm{Br}$ J Clin Pract 1966;20(01):9-13

4 Haider U, Richards P, Gianoukakis AG. Thymic hyperplasia associated with Graves' disease: pathophysiology and proposed management algorithm. Thyroid 2017;27(08):994-1000 